\title{
Self-narrative focus in autobiographical events: The effect of time, emotion, and individual differences
}

\author{
David C. Rubin ${ }^{1,2} \cdot$ Dorthe Berntsen $^{2} \cdot$ Samantha A. Deffler ${ }^{3} \cdot$ Kaitlyn Brodar $^{4}$
}

Published online: 24 August 2018

(C) Psychonomic Society, Inc. 2018

\begin{abstract}
Individuals may take a self-narrative focus on the meaning of personal events in their life story, rather than viewing the events in isolation. Using the Centrality of Event Scale (CES; Berntsen \& Rubin in Behaviour Research and Therapy, 44, 219-231, 2006) as our measure, we investigated self-narrative focus as an individual differences variable in addition to its established role as a measure of individual events. Three studies, with 169, 182, and 190 participants had 11, 10, and 11 different events varied across the dimensions of remembered past versus imagined future, distance from the present, and valence. Imagined future events, events more distant from the present, and positive events all had increased self-narrative focus, in agreement with published theories and findings. Nonetheless, CES ratings for individual events correlated positively with each other within individuals $(r \sim$ .30) and supported a single factor solution. These results are consistent with a stable individual differences tendency toward selfnarrative focus that transcends single events. Thus, self-narrative focus is both a response whereby people relate individual events to their life story and identity and an individual differences variable that is stable over a range of events. The findings are discussed in relation to narrative measures of autobiographical reasoning.
\end{abstract}

Keywords Autobiographical memory · Individual differences · Episodic future thinking · Centrality of Event Scale · Self-continuity

Human beings spend a substantial amount of time mentally traveling away from the present, contemplating their past and future (e.g., Tulving, 1985, 2002). Such mental time travel involves the ability to construct and reexperience or "preexperience" autobiographical events, including their sensory components, spatial setting, and emotional tone. Over the past two decades, researchers have shown that the behavioral and neural structures involved in these constructive processes are highly similar for remembering past events and imagining future events, although some important differences have been

David C. Rubin

david.rubin@duke.edu

1 Department of Psychology and Neuroscience, Duke University, Box 90086, Durham, NC 27708-0086, USA

2 Center on Autobiographical Memory Research, Aarhus University, 8000C Aarhus, Denmark

3 Department of Behavioral Sciences, York College of Pennsylvania, York, PA, USA

4 Department of Psychology, University of Miami, Coral Gables, FL, USA identified (see D'Argembeau, 2012; Rubin, 2014; Schacter et al., 2012; Szpunar, 2010).

Past and future events are not constructed in isolation from other events in an individual's life. During the process of remembering or imagining events, we each consider the implications of each event for other life events, the opening or closing of opportunities, and the importance of the event for our own life story and self-understanding (e.g., Berntsen \& Bohn, 2010; Boucher \& Scoboria, 2014; Conway, Singer, \& Tagini, 2004; Libby \& Eibach, 2011; Singer, Blagov, Berry, \& Oost, 2013). This aspect of the construction of past and future events has been conceptualized as a coherence focus, during which a person steps back and views the event in the context of their overall life story (Boucher \& Scoboria, 2014; Conway et al., 2004; Libby \& Eibach, 2011). Here, we label it selfnarrative focus to underscore that this aspect deals with the broader significance of events in relation to our overall life (e.g., Libby \& Eibach, 2011, p. 720) and not simply the internal coherence of the events as isolated event narratives (Berntsen \& Bohn, 2010; Rubin et al., 2016).

Prior research demonstrates that a self-narrative focus can be induced experimentally, and that such induction changes the qualities of the constructed events (Boucher \& Scoboria, 
2014; Libby \& Eibach, 2011). These findings are important because they demonstrate that a self-narrative focus is not simply determined by the content of the remembered (or imagined) event. Rather, the self-narrative focus can be employed to various degrees by the individual during the process of constructing the event. Accordingly, individuals may differ as to their general inclination to employ such a focus when remembering their past or imagining their future. That is, some people may often consider the role of the event in relation to their life story and identity, whereas others may do so less often. We describe three studies that analyze how a self-narrative focus differs among categories of mental events and whether reliable correlations indicating individual differences nonetheless exist across them.

We use the Centrality of Event Scale (CES; Berntsen \& Rubin, 2006) as a measure of self-narrative focus associated with individual autobiographical events. The CES is widely used. It is cited about 500 times, with about 100 studies reporting results using the scale. We show that, averaged over individuals, self-narrative focus varies systematically with the emotional valence of the remembered or imagined events, regardless of whether the events are contextualized in the past versus future or their temporal distance from the present. Importantly, we also show robust individual differences in the tendency to employ a self-narrative focus, with some individuals more likely to do so than others, irrespective of the type of event. In the following, we first review prior findings on variations in self-narrative focus as a function of other characteristics of the remembered or imagined events. We next review the scarce literature on individual differences in self-narrative focus, viewed as a more general psychometric disposition.

\section{Self-narrative focus and event characteristics}

Several studies have found higher ratings on dimensions equivalent to, or strongly related to, the present conceptualization of self-narrative focus for imagined future events compared with memories of past events. For example, Berntsen and Bohn (2010) asked participants to generate five memories and five future events in response to word cues and to a request for important events. The combination of participants' ratings of two items from the CES and global importance ratings were used to generate an aggregate score for a dimension labelled as self-narrative. For both types of cueing, this measure was higher for future than for past events, which is consistent with other studies showing higher ratings of the personal importance of future compared with past events (e.g., Addis, Wong, \& Schacter, 2008; Boucher \& Scoboria, 2014; D’Argembeau \& Van der Linden, 2004; Newby-Clark \& Ross, 2003) and studies showing higher CES scores for future than for past events (Boucher \& Scoboria, 2014;
Özbek, Bohn, \& Berntsen, 2017; Rasmussen \& Berntsen, 2013; Rubin, 2014). In addition, Berntsen and Bohn (2010) found that the self-narrative scores for all types of events increased systematically with distance in time, consistent with other studies finding higher importance ratings for temporally distant, compared with temporally closer, past and future events (e.g., Addis et al. 2008, D'Argembeau \& Van der Linden, 2004; Özbek et al., 2017).

In addition, a number of studies have compared selfnarrative focus, as measured by the CES, for emotionally positive versus negative autobiographical events and found higher ratings for emotionally positive events (Berntsen, Rubin, \& Siegler, 2011; Rasmussen \& Berntsen, 2013; Rubin, 2014), consistent with the literature on positive selfenhancement (e.g., Ross \& Wilson, 2002). This advantage of positive events has also been found across cultures (Zaragoza Scherman, Salgado, Shao, \& Berntsen, 2015), but is reduced, or abolished, in populations with high symptom levels of posttraumatic stress disorder (PTSD) or depression (Berntsen et al., 2011; Zaragoza Scherman et al., 2015).

\section{Individual differences in self-narrative focus}

Whether or not people adopt a coherence focus in the construction of future and past events is variable. Moreover, it can be manipulated experimentally (Boucher \& Scoboria, 2014; Libby \& Eibach, 2011). This suggests that a self-narrative focus may not be simply a reflection of characteristics of the remembered or imagined event (e.g., whether it is positive or negative). It may also vary as a function of individual dispositions, with some individuals being more likely to use a selfnarrative focus than others.

Several studies have examined individual differences in the styles people adopt when telling about their life experiences (see Adler, Lodi-Smith, Philippe, \& Houle, 2016, for a review). However, surprisingly few studies have examined individual differences in the general processing of autobiographical events using a psychometric measurement approach. One exception is Rubin, Schrauf, and Greenberg (2004), who asked undergraduates to nominate and provide a brief title for 20 distinctive autobiographical memories that could be recalled later from their titles. During the recall phase, participants rated the memories on 17 scales commonly used in autobiographical memory studies, including measures of reliving, belief, emotions, narrative, and sensory systems, as well as ratings of overgeneral memories and the age of the memories. The procedure was repeated at two sessions separated by 2 weeks. The median correlation of the individuals' average rating on each of the 17 scales was.90. One reason for the high correlation could be that Rubin et al. (2004) used the same event at both times, not that people have a general tendency to construct different memories in the same way. To ensure that this was not the case, they correlated the mean of 
10 of the memories with the mean of the other 10 memories. The values, corrected to be comparable with the full set of 20 memories, had a median correlation of.91. Thus, there was considerable stability in the individuals' average ratings on each scale, in which stable individual differences in cognitive style are important (Rubin, 2006).

In a later study, 75 participants diagnosed with PTSD and 45 controls rated word-cued, important, positive, and negative memories (Rubin, Dennis, \& Beckham, 2011). For all four types of memories, patients with PTSD rated self-narrative focus, as measured by ratings of centrality to the life story, higher than did controls. A study with undergraduates who varied in their PTSD symptom severity produced similar results with smaller effects (Rubin, Boals, \& Berntsen, 2008). Relatedly, Berntsen et al. (2011) found a weak positive correlation between the CES as answered for participants' most positive and most traumatic memory, despite substantial mean differences in the ratings of the two types of events. Rubin (2014) reported similar correlations for CES scores for future and past events.

These findings suggest that in addition to the variability explained by the content and types of events (e.g., remembering graduation is likely associated with a greater self-narrative focus than remembering buying strawberries in the grocery store yesterday), additional variability is associated with individual dispositions. That is, some individuals are more prone to adopt a self-narrative focus when thinking of events in their past and future. We designed the present study to examine this issue.

\section{Overview of the present studies}

We use the CES to measure the degree to which the construction of an event involves a self-narrative focus. The CES was devised to measure the integration of a single event, especially a single negatively stressful or traumatic event, into a person's identity and life story (Berntsen \& Rubin, 2006). However, by addressing the extent to which a memory forms a central component of personal identity, a turning point in the life story, and a reference point for everyday inferences (Berntsen \& Rubin, 2006), the CES appears highly suitable for measuring the degree to which people have a self-narrative focus when remembering past and imagining future events. This is consistent with Boucher and Scoboria (2014) who found that an instruction to focus on the broader significance of the event in the person's life led to increased CES scores for the events.

To investigate individual differences in the tendency to employ a self-narrative focus during event construction, we examined the correlations among the CES scores from a range of different events. In Study 1, we examined correlations between the CES for negative events cued by five topics (e.g., relationships among friends or family) and for positive and negative events cued by three timeframes (e.g., during high school). We also examined the stability across sessions of the CES for a single negative event. In Study 2, we extended the emotional valence manipulation in Study 1, by employing a balanced number of positive and negative events. In Study 3, we examined the CES for past and future events that represent similar themes and different lengths of time from the present. The goal of all three studies was to examine how the CES differs among these different event categories and whether reliable correlations indicating individual differences nonetheless exist in people remembering these different categories,.

Consistent with the literature, we expected the CES to be higher for positive than for negative events, and higher for future compared with past events (Berntsen, et al., 2011; Rasmussen \& Berntsen, 2013; Rubin, 2014). We also expected the CES scores to increase as the distance of the events from the present increases. In addition to these differences between events, we expected individual differences in the tendency to adopt a self-narrative focus, so that stable correlations in the CES scores would be found across different event categories.

The statistical approach we used to ensure the reliability of our results was replication. We designed three studies, each with about 180 participants and 10 events. We tested undergraduates in two studies and Amazon Mechanical Turk (MTurk) workers in one. Over the three studies, we used different events to cover either a variety of periods in the participants' lives, or different domains (e.g., school, work, family), or emotional valence, or past versus future events. We chose the number of participants and events with two goals in mind. First, we expected our choice to provide a stable individual difference estimate of self-narrative focus measured by the correlation of CES calculated over the different kinds of events each participant recalled. To foreshadow, we succeeded in this, as the median correlation in the three studies were similar ( $r$ s of .30, .32, and .28). Second, we wanted to have significant differences in the mean ratings of the CES across the dimensions we varied (e.g., emotional valence, past vs. future) to be generally consistent with expectations from the literature. Differences in the mean values of the CES for different kinds of events were needed both to demonstrate that existing theories of the CES were empirically sound and to show that the individual differences stability would occur even when the CES ratings of events varied in important ways. The correlations reported serve as their own effect sizes, and effect sizes were provided for the mean differences tested to ensure a fair evaluation that was independent of the number of participants.

\section{Study 1: Correlations across events and time}

We examined negative and positive events chosen from different domains and time frames to measure the degree of selfnarrative focus. We also examined whether such a focus 
would correlate with the CES and a measure of PTSD symptom severity for a specific traumatic event measured about 3 weeks earlier. For some participants, the specific traumatic event happened to appear as one of their eight negative events, and for others it did not. By using these two subgroups, we could measure the longer-term stability in the CES, and thus self-narrative focus, for the same traumatic event and its general effects on a different traumatic event.

\section{Method}

Participants A total of 169 Duke University undergraduates (121 female, mean age $=18.82$ years, $S D=1.01$, range: $18-22$ years) enrolled in the Department of Psychology and Neuroscience subject pool completed the study.

Materials We used two standardized tests, the seven-item CES (Berntsen \& Rubin, 2004) and the Posttraumatic Stress Disorder Checklist for DSM-5 (PCL-5; Weathers et al., 2013). The CES (Berntsen \& Rubin, 2006) measures the extent to which a memory forms a central component of personal identity, a turning point in the life story, and a reference point for everyday inferences. Among its seven items are: "I feel that this event has become part of my identity." "This event has become a reference point for the way I understand myself and the world." and "This event was a turning point in my life." The PCL-5 has 20 items that match the 20 PTSD symptoms described by the DSM-5 (American Psychiatric Association, 2013). Each item is rated on a 0 to 4 scale of not at all, a little bit, moderately, quite a bit, and extremely, producing a range of 0 to 80 . Among its 20 measures of symptoms are: repeated, disturbing, and unwanted memories of the stressful experience; avoiding memories, thoughts, or feelings related to the stressful experience; loss of interest in activities that you used to enjoy; and having difficulty concentrating.

Procedure At the beginning of each semester, web-based testing is used to screen students for later experiments in the Department of Psychology and Neuroscience subject pool. We included a request for a brief description and dating of the negative event "that bothers you the most," along with the PCL-5 and the CES for that event. We invited all undergraduates who completed our items to participate in the main part of the study, which was also web based.

In all three studies, we fixed the order of the cues to avoid additional error variance from randomization in an individual differences study and to end with positive events to minimize any mood induction that would last beyond the study. In Study 1, we provided cues for participants to generate five negative events based on earlier work with the same population (Rubin, 2014). These were followed by three negative events and three positive events cued by the same timeframes. The resulting 11 cues, in order, were: (1) a negative event related to academics in high school that still affects you a lot, (2) a negative event related to academics at Duke that affects you a lot, (3) a negative event related to death or illness that affects you a lot, (4) a negative event related to relationships among friends or family that affects you a lot, (5) a negative event related to a phone call or message you received that affects you a lot, (6) a negative event that was not already mentioned that occurred before high school that still affects you a lot, (7) a negative event that was not already mentioned that occurred in high school that still affects you a lot, (8) a negative event that was not already mentioned that occurred after high school that affects you a lot, (9) a positive event that occurred before high school that still affects you a lot, (10) a positive event that occurred in high school that still affects you a lot, and (11) a positive event that occurred after high school that affects you a lot.

For each event, participants provided a brief description with an approximate date and completed the CES. The participants then selected without any restriction from among the eight negative memories "the memory that bothers you the most now" and completed the PCL-5 for that memory. Because we had a brief description and the approximate date for the events that bothered the participant the most from both sessions, we could identify those events that were highly likely to be the same or different in both sessions. A research assistant decided whether the screen matched one of the eight negative events. A second scorer helped to decide ambiguous cases. Five participants had descriptions that could not be classified, and one participant failed to complete the PCL-5 in the main session. Of the 169 possible matches, 66 participants chose the same trauma and 98 chose different traumas, including 45 who selected the event used in the screen for one of their events, but not as the event that bothered them the most at the second session. A rescoring of $10 \%$ of the participants by a third scorer produced a $94 \%$ agreement in the original judgments.

\section{Results}

The means, standard deviations, and reliabilities for all measures are shown in Table 1. The reliabilities of the individual tests are all high, ranging from.90 to.96. There was an average of 23.42 days $(S D=5.93$, range: $12-38$ ) between the webbased screen given at the beginning of the semester and the main session - a delay chosen to minimize the effect of the screen on the main session.

Mean differences by event type The means for the CES are shown in Tables 1 and 2. The means for the three negative time-frame events were different and higher than those for the five negative topic events, $F(1,168)=52.58, p<$ $.0001, \eta_{\mathrm{p}}{ }^{2}=.24$. The mean of each of the three positive events was numerically higher than that of each of the eight negative events. To provide a comparison that varied only 
Table 1. Means, standard deviations, and reliabilities for individual events from Study 1

\begin{tabular}{lllll}
\hline Variable & Valence & Mean & SD & $\alpha$ \\
\hline CES event 1, early academics & Negative & 1.80 & 0.84 & .91 \\
CES event 2, later academics & Negative & 1.96 & 0.86 & .90 \\
CES event 3, death, illness & Negative & 3.05 & 1.14 & .94 \\
CES event 4, relationship & Negative & 2.92 & 1.10 & .94 \\
CES event 5, message & Negative & 2.45 & 1.13 & .94 \\
CES event 6, pre-high school & Negative & 2.85 & 1.27 & .96 \\
CES event 7, high school & Negative & 2.80 & 1.19 & .95 \\
CES event 8, post-high school & Negative & 2.80 & 1.16 & .94 \\
CES event 9, pre-high school & Positive & 3.20 & 1.16 & .94 \\
CES event 10, high school & Positive & 3.73 & 1.01 & .93 \\
CES event 11, post-high school & Positive & 3.67 & 1.04 & .93 \\
PCL-5 from most negative of 1 -8 & Negative & 18.03 & 16.47 & .95 \\
CES from screen & Negative & 2.50 & 1.21 & .94 \\
PCL-5 from screen & Negative & 11.16 & 12.02 & .93 \\
\hline
\end{tabular}

Note. $n=169$, except for PCL-5, which is $n=168$

valence, for the next analysis we restricted our comparisons to the cues matched by timeframe.

To examine valence and time frame, we used a 2 (positive vs, negative) $\times 3$ (timeframe) repeated-measures ANOVA. It revealed a significant effect of valence, $F(1,168)=122.18, p$ $<.0001, \eta_{\mathrm{p}}{ }^{2}=.42$, time frame, $F(2,336)=6.61, p<.005, \eta_{\mathrm{p}}^{2}$ $=.04$, and their interaction, $F(2,336)=9.94, p<.0001, \eta_{\mathrm{p}}{ }^{2}=$ .06 . As shown in Table 1, and consistent with published studies (e.g., Berntsen et al., 2011), each of the CES scores was higher for the three positive than for the three negative events from the same categories. The main effect of time frame and the interaction appears to be driven by the pre-high school positive event having a lower mean CES score than the other two positive events, whereas all three negative events had nearly identical mean CES scores.

Correlations among events There are 55 correlations among the CES scores for our 11 events (i.e., the 11 multiplied by $10 /$
2 values in their lower triangular correlation matrix) which are not shown in full but are analyzed in Table 2. All 55 correlations were positive, which is an unlikely chance occurrence. The median correlation was .30. As this correlation was based on 169 participants, it was significant $(p<.0001)$. Except for a .03 and a.08 correlation, the minimum correlation was .14 . The median correlation for the CES scores for the three individual negative events and the individual three positive events is.29 $(p<.001)$. The similarities among the values for the correlations shown in Table 2 supports the claim that the CES can be viewed as a general tendency for individuals to rate events in similar ways. This holds despite substantial differences in the mean value of the CES for individual cues and for groups of cues.

To examine the claim that a single individual differences scale of self-narrative focus could account for the pattern of correlations among the events, the 11 CES scores from each of our 169 participants were analyzed using an exploratory principal factor analysis with squared multiple correlations for the prior communality estimates. If the variables that affected the means shown in Table 1, or other variables that we might be able to discern from examining the factor structure were also important for the correlations among the events, we would expect more than one factor in the analysis. The first five eigenvalues were $3.32,0.47,0.18,0.09$, and 0.07 , supporting a single factor. Given the clear inflection between the first and the remaining eigenvalues, which were all well below 1.00, no rotations were attempted.

\section{Comparisons with the most negative event from the screen} For the 65 participants who reported the same trauma bothered them the most at the screen and during the study, the correlation between their PCL-5 scores was .59 and the correlation between their CES scores was 75 ( $p \mathrm{~s}<.0001)$. Thus, both the CES and PCL-5 were highly correlated over a few weeks when the same event was nominated both times. For the 98 who used different traumas, the correlation between their PCL-5 scores was .66 and the correlation between their CES scores was.44 ( $p s<.0001)$. The CES for different events

Table 2. CES means and correlations from groups of individual events from Study 1

\begin{tabular}{|c|c|c|c|c|c|c|}
\hline \multirow[t]{2}{*}{ Group } & \multirow[t]{2}{*}{ Valence } & \multirow[t]{2}{*}{ Mean } & \multirow[t]{2}{*}{ SD } & \multicolumn{3}{|c|}{$r \mathrm{~s}$ among events } \\
\hline & & & & Median & Min. & Max. \\
\hline All 11 events & Both & 2.84 & 0.61 & .30 & .03 & .45 \\
\hline 5 negative topic events & Negative & 2.44 & 0.69 & .31 & .26 & .45 \\
\hline 3 negative timeframe events & Negative & 2.81 & 0.92 & .39 & .32 & .40 \\
\hline $5 \times 3$ negative events & Negative & 2.58 & 0.46 & .34 & .26 & .43 \\
\hline 3 positive timeframe events & Positive & 3.53 & 0.79 & .30 & .25 & .37 \\
\hline 3 positive $\times 3$ negative events & Both & 3.17 & 0.44 & .29 & .14 & .36 \\
\hline
\end{tabular}

Note. $n=169$ 
correlated within the range of negative events shown in Table 2 . The CES correlation was significantly lower when the comparison was to two different events $(z=3.04, p<.005)$, whereas the PCL-5 correlation was not $(z=-0.64, p=.52)$. Additional study is needed to draw any firm conclusion, but the results are consistent with the view that for extremely negative events, the PCL-5 may be more independent of the particular event being judged than is the CES. The correlations of the time between sessions and the difference in the PCL-5 and CES, as well as the correlations of time with their absolute differences, were not significant (all $p \mathrm{~s}>.29$ ). Thus, variations in the length of the delays we had between the two sessions did not affect the basic results.

The PCL had correlations between participants over a 3week interval that were of the same magnitude as those of the CES, but unlike the CES they were not affected by whether the same or a different negative event was rated. Also in contrast to the CES, the PCL was much higher the second time it was taken. For the first event, participants were simply asked to think of the event that bothered them the most. For the second event, they were asked to generate eight negative events and then to choose from among them the one that bothered them the most. In both cases, events that bothered the participant, not events that were central to their identity, were selected. While this difference did not have a significant effect on the CES, searching for negative memories in eight categories and deciding which was the most troubling may have been the cause of a greater effect on PCL-5 items, which measure troubling reactions to events. Nonetheless, given the speculative nature of this explanation and the large difference in the PCL-5 in the absence of one in the CES, this difference is worth pursuing more systematically in future studies.

\section{Discussion}

We found support for two of our three hypotheses. First, as expected from the literature, the results supported our hypothesis that the CES would be higher for positive than for negative events. Second, although we expected that the CES would increase as the distance of the events from the present increased, the effect of distance from the present did not occur. However, we did not have a simple manipulation of temporal distance but rather confounded temporal distance with content category (e.g., high school). We return to this issue in Study 3. Third, we expected individual differences in the tendency to adopt a self-narrative focus as measured by correlations in the CES scores across different event categories. The 55 correlations among the $11 \mathrm{CES}$ scores had a median of.30 with no clear pattern in the correlations among the various subsets of cues. This finding suggests that rather than needing several measures in addition to self-narrative focus to account for the pattern of correlation, self-narrative focus on its own would suffice, a conclusion supported by finding a single factor in our factor analysis.

The reliabilities for the CES for the individual 11 events were much higher than these correlations, with a range from $\alpha$ $=.91$ to.96. Thus, the general tendency of the CES from one event to predict another accounted for about $10 \%$ of the reliable variance in the CES and did not depend on differences in the valence of the events. In addition, when the same negative event was chosen in the screen and 3 weeks later in the main study, the CES from the two different time points correlated.74, indicating considerable stability for the same event. When two different events were chosen, the correlation was.44, which is lower and within the range of the correlations of the five negative topic events.

\section{Study 2}

We attempt to replicate the basic findings of Study 1 by examining the effects of event valence more directly, using different events and participants drawn from a different and more varied population.

\section{Method}

Participants We paid 200 Amazon MTurk participants \$1.00 each. We excluded eight participants for not providing descriptions for all events, six for using fewer than a total of 300 characters to describe all events, and four for taking less than 8 minutes to complete the task, resulting in 182 participants (130 female, mean age $=35.61, S D=12.07$, median age $=32.5$, range: $18-69$ ). One reported their education as some high school, 16 as a high school diploma or GED, 54 as some college, 14 as an associate's degree, 71 as a bachelor's degree, 16 as a master's degree, and 10 as a doctorate.

Materials We created 10 event cues by preceding the five event categories either by the phrase "an event that is still very emotionally negative related to" or by the phrase "an event that is still very emotionally positive related to." For each event category, the negative cue always immediately preceded the corresponding positive cue. The five event categories included were (1) "school"; (2) "work"; (3) "sports, entertainment, or hobbies" (labeled here as leisure); (4) "a relationship with a family member" (family); (5) "a relationship with someone you know well but who is not a family member" (nonfamily). In addition, in Study 1, all the negative events preceded all the positive events. To ensure that this was not a major factor in our results, in Study 2, we alternated negative and positive events.

Procedure Participants first provided demographic information. After each event cue, they gave a brief description of 
an event, completed the seven-item CES, and provided their age at the time of the event.

\section{Results}

The means, standard deviations, and reliabilities for the CES scores are shown in Table 3 . The reliabilities of the 10 CES tests for individual events are all high, ranging from.91 to.95.

Mean differences by event type As shown in Table 3, and consistent with the published literature and Study 1, the CES scores tended to be higher for the five positive than for the five negative events, which is a replication of Study 1. A 2 (positive vs. negative) $\times$ five (event category) repeated-measures ANOVA revealed a significant effect of valence, $F(1,181)=$ $49.50, \eta_{\mathrm{p}}{ }^{2}=.21$, event category, $F(4,724)=38.45, \eta_{\mathrm{p}}{ }^{2}=.18$, and their interaction, $F(4,724)=8.44, \eta_{\mathrm{p}}{ }^{2}=.04$ (all $p \mathrm{~s}<$ $.0001)$. As shown in Table 3 , the effect of event category and the interaction appears to arise from the CES for the negative family and negative nonfamily being higher than the negative school, work, and leisure categories.

Correlations among events As summarized in Table 4, there are consistent correlations among the 10 mean CES scores for the individual events, supporting the claim that self-narrative focus as measured by the CES can be viewed as an individual differences variable. All 45 correlations $(10 \times 9 / 2)$ are positive. Except for one, they are greater than.191, which, given the sample size, means all but one are significant at the.01 level. As shown in Table 4, the median correlation among all 10 CES scores is.32. This is similar for the correlations among the five negative scores and the five negative event scores correlated with the five positive event scores. The correlation among the five positive event scores is numerically higher at.41, though this was not true in Study 1. The age of the participants, their gender, their approximate years of

Table 3. Means, standard deviations, and reliabilities for individual events from Study 2

\begin{tabular}{lllll}
\hline Variable & Valence & Mean & SD & $\alpha$ \\
\hline Event 1, school & Negative & 2.67 & 1.09 & .91 \\
Event 2, school & Positive & 3.32 & 1.10 & .93 \\
Event 3, work & Negative & 2.83 & 1.07 & .91 \\
Event 4, work & Positive & 3.31 & 1.15 & .94 \\
Event 5, leisure & Negative & 2.52 & 1.15 & .94 \\
Event 6, leisure & Positive & 2.92 & 1.14 & .94 \\
Event 7, family & Negative & 3.55 & 1.18 & .94 \\
Event 8, family & Positive & 3.58 & 1.19 & .95 \\
Event 9, nonfamily & Negative & 3.26 & 1.11 & .93 \\
Event 10, nonfamily & Positive & 3.45 & 1.17 & .94 \\
\hline
\end{tabular}

Note. $n=182$
Table 4 CES means and correlations from groups of individual events from Study 2

\begin{tabular}{llllllll}
\hline \multirow{2}{*}{ Group } & Valence & Mean & SD & \multicolumn{3}{l}{$r$ s among events } \\
\cline { 5 - 8 } & & & & & Median & Min. & Max. \\
\hline All 10 events & Both & 3.14 & 0.71 & .32 & .12 & .50 \\
5 negative events & Negative & 2.96 & 0.75 & .32 & .23 & .39 \\
5 positive events & Positive & 3.32 & 0.82 & .41 & .20 & .50 \\
5 negative $\times 5$ positive & Both & 3.14 & 0.71 & .31 & .12 & .47 \\
\hline
\end{tabular}

Note. $n=182$

education, and the time taken to complete the task did not correlate significantly with the average CES scores for either the five negative, five positive, or all 10 combined events (maximum correlation of.09).

To examine the claim that a single individual differences scale of self-narrative focus could account for the pattern of the correlations among the events, the 10 CES scores from each of our 182 participants were analyzed using a principal factor analysis as was done in Study 1. The first five eigenvalues were $3.32,0.33,0.28,0.17$, and 0.07 , replicating the support found for a single factor in Study 1.

\section{Discussion}

We found support for our two hypotheses. First, as predicted and in congruence with Study 1, the CES was higher for positive than for negative events. Second, we expected individual differences in the tendency to adopt a self-narrative focus, as measured by correlations in the CES scores across different event categories. The reliabilities for the CES for the individual 10 events ranged from $\alpha=.91$ to .95 . The 45 correlations among the 10 CES scores had a median of.32. These findings replicate the findings from Study 1, where the range of $\alpha$ s was .91 to.96 and the median correlation of all 11 events was.30. Thus, as in Study 1, the general tendency of the CES from one event to predict another accounted for about $10 \%$ of the reliable variance in the CES. However, unlike Study 1, the correlation depended on differences in the valence of the events. Nonetheless, as in Study 1, the correlations produced a single factor solution supporting a single underlying dimension of self-narrative focus.

\section{Study 3}

We include imagined future events because future events are less constrained by actual occurrences and thus might give a clearer description of the tendency to adopt a self-narrative focus. We used time-frame cues for both past and future events, instead of the topic cues of Study 2. This provided 
more neutral cues in terms of content, emphasized time into the future and past, and allowed us to use existing studies with this temporal dimension in interpreting our results (e.g., Berntsen \& Bohn, 2010; D’Argembeau \& Van der Linden, 2004; Spreng \& Levine, 2006; Szpunar \& McDermott, 2008). After these cues, we asked for an extremely negative and an extremely positive event to attempt a replication of the valence effects found in the earlier studies.

\section{Method}

Participants We paid 200 Amazon MTurk participants \$2.00 each and prevented individuals who had taken part in Study 2 from participating based on their MTurk IDs. We removed 10 participants for not completing the study or for completing it in less than 8 minutes, resulting in 190 participants ( 83 female, mean age $=35.46, S D=10.91$, median age $=33$, range: 19 75). Seventeen reported their education level as a high school diploma or GED, 40 as some college, 23 as an associate's degree, 72 as a bachelor's degree, 32 as a master's degree, and seven as a doctorate.

Materials We created four event cues by using the phrase "please think of an event you remember that occurred" followed by one of the following four timeframes: (1) "within the last week, but not today"; (2) "between a week and a month ago"; (3) "between a month and a year ago"; and (4) "more than 1 year ago." These preceded four more event cues that we created by using the phrase "please think of an event that might occur" followed by the same periods but with minor adjustments to indicate the future. Each of these eight had a CES. Next, there was a CES and PCL-5 for "the event that currently bothers you the most" (referred to as the negative event). Finally, there was a CES for "an event for which you are happy, proud, or thankful" (referred to as the positive event).

Procedure Participants provided a brief description for all events after reading the event cues before they proceeded to the rest of the study. For the past and future events longer than 1 year and the negative and the positive event, participants indicated their age at the event. For the past and future events only, participants next completed a valence measure that began "while remembering the event, my emotions are" and used a scale ranging from extremely negative $(-3)$ to extremely positive (3). After providing these ratings, participants completed the CES for each event. At the end of the study, they provided demographic information.

\section{Results}

The means, standard deviations, and reliabilities for the CES scores are shown in Table 5. The reliabilities of the individual tests are all high, ranging from.92 to.95. The age of the participants, their gender, their approximate years of education, and the time taken to complete the task correlated in sporadic ways with the average CES scores. We report these for completeness, but as correlations with these four variables were not significant in Study 2, we cannot claim they would occur in a replication. For the 10 event types, three correlations were significant at the $p<.05$ level, uncorrected for multiple comparisons. Age and the CES for the positive event correlated .15 ; gender and the CES for the event that occurred within the last week correlated -.19, indicating that women had lower scores; and time taken and the CES for the event that occurred in the last month correlated.17.

Mean differences by event type We analyzed both the CES scores and the emotional valence in a 2 (past vs. future) $\times 4$ (time frame) repeated-measures ANOVA. For the CES, there were main effects of past versus future, $F(1,189)=5.60, p<$ $.05, \eta_{\mathrm{p}}{ }^{2}=.03$, and time frame, $F(3,567)=201.75, p<.0001$, $\eta_{\mathrm{p}}{ }^{2}=.52$, but not their interaction, $F(3,567)=1.21, p=.3058$, $\eta_{\mathrm{p}}{ }^{2}=.01$. As can be seen in the means in Table 5, the CES means for the future are always numerically greater than the means from the past, and in both the past and the future there is a monotonic increase in means as the time from the present increases into either the future or the past. To investigate these effects further, we divided the responses from the more-than-

Table 5 Means, standard deviations, and reliabilities for individual events from Study 3

\begin{tabular}{lllll}
\hline Variable & Past/ & Mean & SD & $\alpha$ \\
& Future & & & \\
\hline CES within week & Past & 2.58 & 1.11 & .93 \\
CES within month & Past & 2.80 & 1.22 & .94 \\
CES within year & Past & 3.56 & 1.13 & .93 \\
CES over a year & Past & 3.99 & 0.96 & .92 \\
CES within week & Future & 2.60 & 1.24 & .95 \\
CES within month & Future & 2.86 & 1.23 & .95 \\
CES within year & Future & 3.75 & 1.08 & .94 \\
CES over a year & Future & 4.19 & 0.93 & .94 \\
CES negative event & Past & 3.66 & 0.99 & .92 \\
CES positive event & Past & 4.13 & 0.95 & .95 \\
PCL negative event & Past & 1.90 & 0.84 & .95 \\
Valence within week & Past & 1.10 & 1.97 & \\
Valence within month & Past & 1.12 & 2.07 & \\
Valence within year & Past & 1.11 & 2.20 & \\
Valence more than a year & Past & 0.83 & 2.26 & \\
Valence within week & Future & 1.46 & 1.68 & \\
Valence within month & Future & 1.61 & 1.58 & \\
Valence within year & Future & 1.97 & 1.46 & \\
Valence more than a year & Future & 2.17 & 1.46 & \\
\hline
\end{tabular}

Note. $n=190$ 
a-year-cue category into three divisions that provided a nearly equal number of responses in each category: within the first year, within the second and third year, and more than 3 years ago. For the past, the means were $3.70,3.91$, and 4.24, $F(1$, $188)=9.98, p<.005, \eta_{\mathrm{p}}{ }^{2}=.05$. For the future, the means were $3.89,4.36$, and 4.30, $F(1,188)=6.28, p<.05, \eta_{\mathrm{p}}{ }^{2}=.03$. As shown in Fig. 1, both the past and the future continued the trend shown for the cued categories in Table 5.

For emotional valance, there was a main effect of past versus future, $F(1,189)=47.95, p<.0001, \eta_{\mathrm{p}}{ }^{2}=.20$, but not for time frame, $F(3,567)=2.02, p=.1098, \eta_{\mathrm{p}}{ }^{2}=.01$; the interaction was significant, $F(3,567)=7.12, p<.0001, \eta_{\mathrm{p}}{ }^{2}=$ .04 . As can be seen in the means of emotional valence in Table 5 , the means for the future are always more positive than the means from the past. This is a standard finding for events requested without specific details about content (Berntsen \& Bohn, 2010; D’Argembeau \& Van der Linden, 2006; Finnbogadóttir \& Berntsen, 2013; Newby-Clark \& Ross, 2003; Rubin, 2014). The interaction appears to arise from the difference between the past and the future valence, with the future but not the past monotonically increasing, producing mean differences of.36, .49, .86, and 1.34 as the temporal distance from the present increases (Grysman, Prabhakar, Anglin, \& Hudson, 2013).

In addition to the comparison of past and future memories, we cued one negative and one positive event. As shown in Table 5, and, consistent with the literature and the two previous studies, the positive memories had a higher CES score than the negative one (4.13 vs. 3.66), $F(1,189)=25.53, p<$ $.0001, \eta_{\mathrm{p}}{ }^{2}=.12$.

Correlations among events As shown in Table 6, there are reasonable correlations among the mean CES scores for the four past, four future, negative, and positive events, supporting the claim that the tendency to adopt a selfnarrative focus varies across individuals. All 45 correlations among the 10 CES measures are positive. For the 28

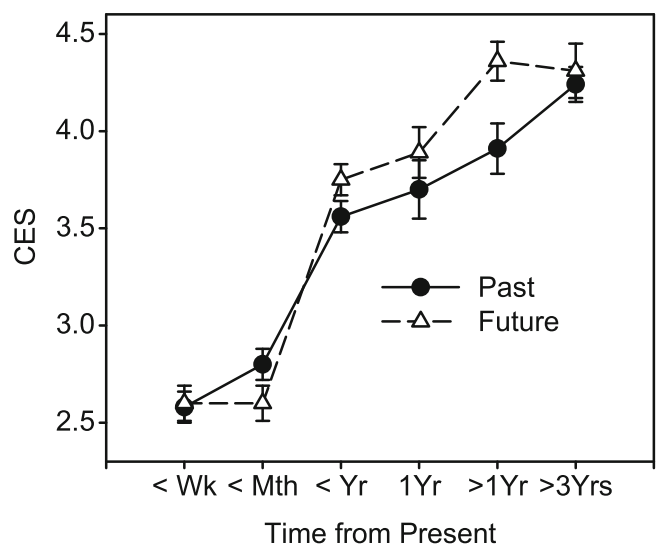

Fig. 1 Mean value of the CES as a function of time from the present. Error bars are standard errors
Table 6 CES Means and correlations from groups of individual events from Study 3 .

\begin{tabular}{llllll}
\hline \multirow{2}{*}{ Group } & Mean & SD & \multicolumn{3}{l}{$r$ s among events } \\
\cline { 4 - 7 } & & & Median & Min. & Max. \\
\hline All 10 events & 3.41 & 0.63 & .28 & .00 & .50 \\
All 8 past and future events & 3.29 & 0.73 & .36 & .08 & .50 \\
4 past events & 3.23 & 0.80 & .35 & .21 & .50 \\
4 future events & 3.35 & 0.81 & .37 & .24 & .44 \\
Past $\times$ Future events & 3.29 & 0.97 & .34 & .08 & .45 \\
\hline
\end{tabular}

Note. $n=190$

correlations among the past and future events, all except for two are greater than.21, which, given the sample size, means all but two are significant at the $p<.01$ level. The median correlations for all $10 \mathrm{CES}$ scores was .28. For just the eight past and future events the median correlations was .36. The correlation between the single positive and negative event was.14, $p<.05$, which is at the low end of the range observed in the first two studies for correlations of individual positive and negative events.

To examine the claim that a single individual differences scale of self-narrative focus could account for the correlations among the events, the 10 CES scores from each of our 190 participants were analyzed using a principal factor analysis as was done in Studies 1 and 2. The first five eigenvalues were $2.85,0.51,0.16,0.13$, and 0.06 , supporting a single factor and replicating Studies 1 and 2.

\section{Discussion}

All four hypotheses were supported. First, the CES was higher for positive than for negative events. Second, the CES was higher for future than past events. Third, the CES increased as the distance of the events from the present increased. Fourth, we found individual differences in the tendency to adopt a self-narrative focus as measured by correlations in the CES scores across different event categories.

The reliabilities for the CES for the individual 10 events ranged from $\alpha=.92$ to. 95 . The 45 correlations among the 10 CES scores had a median of.28. These findings replicate the findings from Studies 1 and 2 in terms of the range of $\alpha$ s and the median correlation among all events, and the valence effect on the mean CES scores. As in those studies, the general tendency of the CES from one event to predict another accounted for about $10 \%$ of the reliable variance. Moreover, the correlations among the CES did not vary systematically with whether the events were in the past or future, producing a single factor solution.

The increase in self-narrative focus as time increases into either the future or the past is a replication of past findings, but 
it is also an extension. In the same study, the increase holds both for experimenter-selected cued time frames and for memories from the same cue that were grouped into time frames based on the dates given by the participants. This eliminates two possible explanations of the effect that holds only for the cued timeframes. The first is that the request for times in the distant past and future implies to the participants that the events should be more important to them and therefore higher on the CES. The second is that the increasing size of the cued timeframes from a week to all the participants' past and expected future life that is greater than a year from the present allows for events more important to the participants to be retrieved. See Berntsen and Bohn (2010) for similar findings for freely generated future and past events.

\section{General discussion}

When remembering or imagining a personal event, individuals can have a self-narrative focus for the event. That is, they can focus on the role of the event in their life story and identity rather than on the event viewed in isolation (e.g., Berntsen \& Bohn, 2010; Boucher \& Scoboria, 2014; Conway et al., 2004; Libby \& Eibach, 2011; Prebble, Addis, \& Tippett, 2013). We used the Centrality of Event Scale (CES) to measure selfnarrative focus across systematically varied types of remembered and imagined events. Across three studies, the tendency spontaneously to adopt a self-narrative focus when constructing personal events varied systematically with the emotional valence of the events, their temporal distance from the present, and whether the events were in the past or future. These findings replicated and extended earlier findings on ways in which the properties of events affect the degree of self-narrative focus as measured by the CES. In addition, across these different categories of events, we also demonstrated robust individual differences in the tendency to adopt a self-narrative focus, with some individuals doing so to a greater extent than others. These positive correlations between the CES ratings across event categories suggest that some of the variance in this measure reflects stable individual dispositions. Thus, the extent to which a self-narrative focus is adopted depends on two interacting factors: characteristics of the remembered or imagined event and dispositions of the individual. In the following, we discuss these findings in greater detail.

Studies 1 and 2 demonstrated that emotionally positive events were associated with higher levels of self-narrative focus. This is consistent with previous work showing that positive events are generally viewed as more central to one's life story and identity compared with negative events. For example, Rasmussen and Berntsen (2013) had students generate positive and negative personal events that were dated in either their past or their future and then rate the events on the CES. Across both temporal directions, participants rated positive events as more central than negative events. Berntsen et al. (2011) reported similar findings in a large sample of older adults who completed the CES for memories of past events identified as either highly stressful or highly positive. The participants viewed positive memories as markedly more central to life story and identity compared with the memories of the stressful events, except in individuals with high levels of PTSD symptoms, for whom the centrality of the stressful events approached, and in some cases exceeded, the centrality level of the positive events. Similar findings were reported by Zaragoza Scherman et al. (2015) across four different cultures.

The fact that individuals in general are more inclined to adopt a self-narrative focus for positive events than for negative events may reflect a positive self-enhancement bias, akin to the self-enhancement biases in memory observed by social psychologists (Ross \& Wilson, 2002; Sedikides \& Gregg, 2008; Wilson \& Ross, 2001). For example, people feel temporally closer to events viewed as favorable to their present self-image, compared with past events that may hold negative implications for their current self-view (Ross \& Wilson, 2002). In the same way, viewing positive events as central to one's life story and identity may serve to support a positive view of oneself. Relatedly, the fact that the intensity of positive events generally fades more slowly over time than the intensity of negative events (i.e., the fading affect bias: see Ritchie et al., 2006, for a review) may be associated with an enhanced self-narrative focus of positive events, for example, by increasing the sense of self-continuity associated with emotionally positive events (Ritchie, Sedikides, \& Skowronski, 2015). In addition, culturally shared norms predict positive life events to be part of the life story (Berntsen \& Rubin, 2004), which also is likely to contribute to the enhanced self-narrative focus of positive life events (see Berntsen et al., 2011, for further discussions).

Study 3 showed that future events were associated with a greater self-narrative focus than were past events. This is consistent with previous studies in which participants rated the CES, or parts of it, for both types of mental events (Berntsen \& Bohn, 2010; Ottsen \& Berntsen, 2015; Özbek et al., 2017; Rasmussen \& Berntsen, 2013; Rubin, 2014). Exceptions are studies involving involuntarily generated memories/images of past and future events, which do not find a higher selfnarrative focus for future, relative to past, events (Berntsen \& Jacobsen, 2008; Cole, Staugaard, \& Berntsen, 2016; Finnbogadóttir \& Berntsen, 2011). This may be because these studies examined mental events generated with little involvement of the top-down and schema-based construction that may be responsible for the differences between past and future events observed in other studies that used voluntary retrieval in response to generic cues (Berntsen \& Bohn, 2010; Rubin, 2014). Study 3 also showed a steady increase in a selfnarrative focus with increasing distance from the present for 
both past and future events, replicating and extending previous work (Berntsen \& Bohn, 2010; Özbek et al., 2017). This finding is consistent with temporal construal theory (Trope \& Liberman, 2003), according to which people form more abstract representations (high-level construals) of temporally distant events and more concrete representations (low-level construals) of temporally close events. Since self-narrative focus is concerned with the role of the event in the context of the life story, it likely involves the high-level construal of extracting the essential information about an event (e.g., having a child) in contrast to low-level construal including concrete, contextual, and accidental details (e.g., changing diapers). It therefore makes sense that the likelihood of adopting a self-narrative focus increases with temporal distance from the present (e.g., Berntsen \& Bohn, 2010).

In this research, we measured each individual's selfnarrative focus by averaging the scores of the seven-item CES for events intended to sample autobiographical memory. Another technique for future research would be to choose events that are especially relevant to the research question being pursued, such as a larger set of extremely negative and positive events (e.g., Berntsen et al., 2011), as past research has often only used one or a few events of each type. Future research might also examine individual differences in selfnarrative focus as a function of the narrative themes of the events, such as agency versus communion and redemption versus contamination (e.g., Adler et al., 2016).

In spite of these highly systematic differences between events in the degree to which they were associated with a self-narrative focus, we found robust positive correlations between the ratings across different events, which produced a single factor solution in all three studies. This suggests individual differences in autobiographical remembering and imagining style with some individuals showing a greater tendency for self-narrative focus irrespective of types of autobiographical events. This can be seen as consistent with studies showing robust individual differences in the ways individuals construe narratives about experiences in their life (see Adler et al., 2016, for a review). Because self-narrative focus, as defined here, measures the extent to which individuals perceive personal events as central to their life story and identity, it may be seen as a psychometric measure of a tendency to engage in autobiographical reasoning - that is, a "dimension of narrative focused on the subjective impact of the event on the self" (McLean, Pasupathi, Greenhot, \& Fivush, 2017, p. 57; see Habermas \& Bluck, 2000). In other words, the consistent individual differences in self-narrative focus we have demonstrated here may be related to individual differences in autobiographical reasoning as observed in narratives, such as individual differences in the tendency to make statements about the impact of life experiences on the sense of self when telling personal-event stories (McLean et al., 2017). Recent research has shown that such meaning-making statements are a central component of narrative coherence (e.g., Adler, Waters, Poh, \& Seitz, 2018). Future research should explore the relation between self-narrative focus as operationalized here and narrative measures of meaning making. More broadly, future research should resolve how individual differences in selfnarrative focus correlate with other individual dispositions, such as measures of personality traits.

Examining individual differences in self-narrative focus is also relevant to understanding clinical disorders. For example, with respect to mechanisms underlying PTSD, Boucher and Scoboria (2014) demonstrated experimentally that adopting a self-narrative focus is associated with perceiving more psychological and material impact of the event (relative to taking an experiential-oriented focus). Speculatively, this might suggest that a general tendency to adopt such a focus is a vulnerability factor for developing PTSD after exposure to negative, stressful events, since such focus would amplify the importance of the event and thus likely work against disengagement and inhibition of negative thoughts related to the event. This suggestion agrees with Rubin et al. (2011) who compared PTSD patients and controls and found that a single measure of life story relatedness was rated higher in the PTSD group for a range of different autobiographical events, including, but not limited to, the primary traumatic event.

Taking a self-narrative focus requires an ability to make connections between the self and individual events. One aspect of impaired self-continuity is difficulties with making such connections. However, although schizophrenia is a disorder associated with impoverished self-continuity, Allé et al. (2016) found no differences between patients with schizophrenia and controls when they were asked to rate the CES for personally important memories and imagined future events. Holm, Thomsen, and Bliksted (2016) reported similar findings, using a single item measure of centrality in patients with schizophrenia versus controls. Yet, within the patient group, Holm et al. (2016) reported a negative correlation between centrality ratings of life-story chapters and positive symptoms of schizophrenia, suggesting that patients with more severe symptom levels were less likely to adopt a selfnarrative focus in relation to autobiographical events. However, these findings should be interpreted with caution due to a small sample size. More research is needed to understand self-narrative focus in relation to symptoms of schizophrenia.

In conclusion, a self-narrative focus as measured by the CES varies systematically as a function of characteristics of remembered or imagined events, with positive events, events dated in the personal future, and events distant to the present more strongly associated with such a focus. In addition to these systematic event-related differences, we found robust positive correlations between CES ratings across event categories, showing that some individuals are more inclined than others to adopt a self-narrative focus, irrespective of 
characteristics of the remembered or imagined event. Future research should examine how this tendency correlates with measures of other individual dispositions, such as measures of well-being and rumination, and individual differences in autobiographical reasoning.

Author note We wish to thank Rick Hoyle for statistical advice.

Funding The authors disclosed receipt of the following financial support for the research, authorship, and/or publication of this article: Preparation of this manuscript was supported by Grant DNRF89 from the Danish National Research Foundation and Grant R01 MH066079 from the National Institute of Mental Health.

\section{Compliance with ethical standards}

Conflict interest The authors declared no potential conflicts of interest with respect to the research, authorship, and/or publication of this article.

\section{References}

Addis, D. R., Wong, A. T., \& Schacter, D. L. (2008). Age-related changes in the episodic simulation of future events. Psychological Science, $19,33-41$.

Adler, J. M., Lodi-Smith, J., Philippe, F. L., \& Houle, I. (2016). The incremental validity of narrative identity in predicting well-being: A review of the field and recommendations for the future. Personality and Social Psychology Review, 20, 142-175.

Adler, J. M., Waters, T. E. A., Poh, J., \& Seitz, S. (2018). The nature of narrative coherence: An empirical approach. Journal of Research in Personality, 74, 30-34.

Allé, M. C., D’Argembeau, A., Schneider, P., Potheegadoo, J., Coutelle, R., Danion, J.-M., \& Berna, F. (2016). Selfcontinuity across time in schizophrenia: An exploration of phenomenological and narrative continuity in the past and future. Comprehensive Psychiatry, 69, 53-61.

American Psychiatric Association. (2013). Diagnostic and statistical manual of mental disorders (5th ed.).. Washington, DC: Author.

Berntsen, D., \& Bohn, A. (2010). Remembering and forecasting: The relation between autobiographical memory and episodic future thinking. Memory \& Cognition, 38, 265-278.

Berntsen, D., \& Jacobsen, A. S. (2008). Involuntary (spontaneous) mental time travel into the past and future. Consciousness and Cognition, 17, 1093-1104.

Berntsen, D., \& Rubin, D. C. (2004). Cultural life scripts structure recall from autobiographical memory. Memory \& Cognition, 32, 427-442.

Berntsen, D., \& Rubin, D. C. (2006). Centrality of Event Scale: A measure of integrating a trauma into one's identity and its relation to post-traumatic stress disorder symptoms. Behaviour Research and Therapy, 44, 219-231.

Berntsen, D., Rubin, D. C., \& Siegler, I. C. (2011). Two versions of life: Emotionally negative and positive life events have different roles in the organization of life story and identity. Emotion, 11, 1190-1201.

Boucher, C. M., \& Scoboria, A. (2014). Reappraising past and future transitional events: The effects of mental focus on present perceptions of personal impact and self-relevance. Journal of Personality, $83,361-375$.

Cole, S. N., Staugaard, S. R., \& Berntsen, D. (2016). Inducing involuntary and voluntary mental time travel using a laboratory paradigm. Memory \& Cognition, 44, 376-389.
Conway, M. A., Singer, J. A., \& Tagini, A. (2004). The self and autobiographical memory: Correspondence and coherence. Social Cognition, 22, 491-529.

D'Argembeau, A. (2012). Autobiographical memeory and future thinking. In D. Berntsen \& D. C. Rubin (Eds.), Understanding autobiographical memory: Theories and approaches. (pp. 311-330). Cambridge, UK: Cambridge University Press

D'Argembeau, A., \& Van der Linden, M. (2004). Phenomenal characteristics associated with projecting oneself back into the past and forward into the future: Influences of valence and temporal distances. Consciousness and Cognition, 13, 844-858.

D'Argembeau, A., \& Van der Linden, M. (2006). Individual differences in the phenomenology of mental time travel: The effect of vivid visual imagery and emotion regulation strategies. Consciousness and Cognition, 15, 342-350.

Finnbogadóttir, H., \& Berntsen, D. (2011). Involuntary mental time travel in high and low worriers. Memory, 19, 625-640.

Finnbogadóttir, H., \& Berntsen, D. (2013). Involuntary future projections are as frequent as involuntary memories, but more positive. Consciousness and Cognition, 22, 272-280.

Grysman, A., Prabhakar, J., Anglin, S. M., \& Hudson, J. A. (2013). The time travelling self: Comparing self and other in narratives of past and future events. Consciousness and Cognition, 22, $742-755$.

Habermas, T., \& Bluck, S. (2000). Getting a life: The emergence of the life story in adolescence. Psychological Bulletin, 126, 748-769.

Holm, T., Thomsen, D. K., \& Bliksted, V. (2016). Life story chapters and narrative self-continuity in patients with schizophrenia. Consciousness and Cognition, 45, 60-74.

Libby, L. K., \& Eibach, R. P. (2011). Self-enhancement or self-coherence? Why people shift visual perspective in mental images of the personal past and future. Personality and Social Psychology Bulletin, 37, 714-726.

McLean, K. C., Pasupathi, M., Greenhoot, A. F., \& Fivush, R. (2017). Does intra-individual variability in narration matter and for what? Journal of Research in Personality, 69, 55-66.

Newby-Clark, I. R., \& Ross, M. (2003). Conceiving the past and future. Personality and Social Psychology Bulletin, 29, 807-818.

Ottsen, C. L., \& Berntsen, D. (2015). Prescribed journeys through life: Cultural differences in mental time travel between Middle Easterners and Scandinavians. Consciousness and Cognition, 37, $180-193$.

Özbek, M., Bohn, A., \& Berntsen, D. (2017). Imagining the personal past: Episodic counterfactuals compared to episodic memories and episodic future projections. Memory \& Cognition, 45, 375-389.

Prebble, S. C., Addis, D. R., \& Tippett, L. J. (2013). Autobiographical memory and sense of self. Psychological Bulletin, 139, 815-840.

Rasmussen, A. S., \& Berntsen, D. (2013). The reality of the past versus the ideality of the future: Emotional valence and functional differences between past and future mental time travel. Memory \& Cognition, 41, 187-200.

Ritchie, T. D., Sedikides, C., \& Skowronski, J. J. (2015). Emotions experienced at event recall and the self: Implications for the regulation of self-esteem, self-continuity, and meaningfulness. Memory, 24, 577-591.

Ritchie, T. D., Skowronski, J. J., Wood, S. E., Walker, W. R., Vogl, R. J., \& Gibbons, J. A. (2006). Event self-importance, event rehearsal, and the fading affect bias in autobiographical memory. Self and Identity, $5,172-195$.

Ross, M., \& Wilson, A. E. (2002). It feels like yesterday: Self-esteem, valence of personal past experiences, and judgments of subjective distance. Journal of Personality and Social Psychology, 82, 792803.

Rubin, D. C. (2006). The basic-systems model of episodic memory. Perspectives on Psychological Science, 1, 277-311. 
Rubin, D. C. (2014). Schema driven construction of future autobiographical traumatic events: The future is much more troubling than the past. Journal of Experimental Psychology: General, 143(2), 612-630.

Rubin, D. C., Boals, A., \& Berntsen, D. (2008). Memory in posttraumatic stress disorder: Properties of voluntary and involuntary, traumatic and non-traumatic autobiographical memories in people with and without PTSD symptoms. Journal of Experimental Psychology: General, 137, 591-614.

Rubin, D. C, Deffler, S. A., Ogle, C. M., Dowell, N., Graesser, A. C., \& Beckham, J. C. (2016). Participant, rater, and computer measures of coherence in posttraumatic stress disorder. Journal of Abnormal Psychology, 125, 11-25.

Rubin, D. C., Dennis, M. F., \& Beckham, J. C. (2011). Autobiographical memory for stressful events: The role of autobiographical memory in posttraumatic stress disorder. Consciousness and Cognition, 20, 840-856.

Rubin, D. C., Schrauf, R. W., \& Greenberg, D. L. (2004). Stability in autobiographical memories. Memory, 12, 712-721.

Schacter, D. L., Addis, D. R., Hassabis, D., Martin, V. C., Spreng, R. N., \& Szpunar, K. K. (2012). The future of memory: Remembering, imagining, and the brain. Neuron, 76, 677-694.

Sedikides, C., \& Gregg, A. P. (2008). Self-enhancement: Food for thought. Perspectives on Psychological Science, 3, 102-116.

Singer, J. A., Blagov, P., Berry, M., \& Oost, K. M. (2013). Self-defining memories, scripts, and the life story: Narrative identity in personality and psychotherapy. Journal of Personality, 81, 569-582.
Spreng, R. N., \& Levine, B. (2006). The temporal distribution of past and future autobiographical events across the lifespan. Memory \& Cognition, 34, 1644-1651.

Szpunar, K. K. (2010). Episodic future thought: An emerging concept. Perspectives on Psychological Science, 5, 142-162.

Szpunar, K. K., \& McDermott, K. B. (2008). Episodic future thought and its relation to remembering: Evidence from ratings of subjective experience. Consciousness and Cognition, 17, 330-334.

Trope, Y., \& Liberman, N. (2003). Temporal construal. Psychological Review, 110, 403-421.

Tulving, E. (1985). Memory and consciousness. Canadian Psychology, 26, 1-26.

Tulving, E. (2002). Episodic memory: From mind to brain. Annual Review of Psychology, 53, 1-25.

Weathers, F. W., Litz, B. T., Keane, T. M., Palmieri, P. A., Marx, B. P., \& Schnurr, P. P. (2013). The PTSD Checklist for DSM-5 (PCL-5). Scale available from the National Center for PTSD: www.ptsd.va.gov

Wilson, A. E., \& Ross, M. (2001). From chump to champ: People's appraisals of their earlier and present selves. Journal of Personality and Social Psychology, 80, 572-584.

Zaragoza Scherman, A., Salgado, S., Shao, Z., \& Berntsen, D. (2015). Event centrality of positive and negative autobiographical memories to identity and life story across cultures. Memory, 23, 1152-1171. 